\title{
ANÁLISIS INTERSECCIONAL Y ENFOQUE INTERCULTURAL EN EL ESTUDIO DE LA CIUDADANÍA Y LA PARTICIPACIÓN. CONSIDERACIONES EPISTEMOLÓGICAS
}

\author{
INTERSECTIONALITY AND INTERCULTURAL APPROACH IN THE STUDY OF \\ CITIZENSHIP AND PARTICIPATION. EPISTEMOLOGICAL CONSIDERATIONS
}

\author{
Héctor Sánchez-Melero* e Inés Gil-Jaurena*
}

\begin{abstract}
El estudio de la práctica ciudadana requiere del acercamiento a una realidad social marcada por la desigualdad (histórica y contemporánea) en el ejercicio de la ciudadanía, y por el dinamismo y la transformación con que se desarrolla la participación ciudadana en la actualidad. En este artículo se plantean algunas consideraciones epistemológicas en torno a la diversidad y los mecanismos de categorización social que influyen en la práctica ciudadana desigual. Se apuesta por abordar la complejidad del tema desde dos enfoques complementarios, la interseccionalidad y el enfoque intercultural, entendidos desde una perspectiva crítica.
\end{abstract}

Palabras clave: ciudadanía, diversidad, intercultural, interseccionalidad, participación.

The study of active citizenship requires to approach a social reality marked by inequality ( historical and contemporary) in the practice of citizenship, and by the dynamism and transformation that characterizes public participation that takes place today. This paper discusses some epistemological considerations about diversity and social categorization mechanisms that influence unequal citizenship practices. It is proposed to address the complexity of the subject from two complementary approaches, intersectionality and intercultural approach, understood from a critical perspective .

Key words: citizenship, diversity, interculturalism, intersectionality, participation.

\section{Introducción: Sobre ciudadanía y participación}

Desde que Marshall ([1949]1997) ${ }^{1}$ planteara el surgimiento de la ciudadanía como un proceso unido al desarrollo de los diversos derechos propios de los Estados democráticos, muchos de los debates sobre este concepto y las críticas al mismo han tenido en cuenta esta relación entre ciudadanía y Estado, relación mediatizada por los derechos que se le reconocen. Ya fuera el Estado liberal o el posterior Estado del bienestar, la ciudadanía ha venido considerándose unida al principio de igualdad, principio de concepción universalista, pero cuya concreción real, al relacionarse con la identidad nacional o con la relación de pertenencia a una comunidad política, sirvió para excluir a extensos grupos de población. Por tanto, como escribe Babiano "la historia de la ciudadanía es tanto la historia de la extensión de los derechos que lleva consigo cuanto la historia de la exclusión del propio estatus de ciudadanía y del acceso a tales derechos" (citado en Cabrera, 2002:91); de este modo la ciudadanía no sería un principio universalista, sino diferenciador (Irurozqui, 2008).

En sus inicios ilustrados, la condición de ciudadano estaba reservada a los sujetos considerados autónomos y racionales, lo que dejaba fuera del principio de igualdad a los clasificados jurídicamente como "dependientes", es decir, las mujeres, los no propietarios y, más allá de las fronteras nacionales, los sujetos colonizados (Suárez Navaz, 1998). Por ello, los debates y críticas posteriores sobre la ciudadanía, producidas por diversas corrientes de pensamiento, trataban de incluir a estos colectivos excluidos, a la vez que desvelaban los mecanismos que permitían esta exclusión y proponían distintas estrategias para superarlos, llegando incluso a cuestionar los fundamentos sobre los que se construía el concepto de ciudadanía (Pateman, 1996; Young, 1996; Kimlincka, 1999; Benhabib, 2006; Amorós, 2008).

Actualmente sin embargo, la ciudadanía está sufriendo una amplia transformación. Por un lado, su aplicación práctica tal y como la conocemos parece estar amenazada por los procesos de globalización * Universidad Nacional de Educación a Distancia (UNED), España. Correos electrónicos: hsanchez@invi.uned.es; inesgj@edu.
uned.es 
al tiempo que se amenazan los derechos sobre los que se sustenta, la crisis del Estado del bienestar está poniendo en peligro los derechos sociales y políticos y en última instancia hasta los derechos civiles en distintas regiones del planeta ${ }^{3}$. A su vez, la existencia de derechos de pretensión universalista como los derechos humanos podrían suponer el surgimiento de una ciudadanía de carácter supranacional (Sassen, 2001), a lo que hay que añadir la aparición de derechos de tercera generación, que superan la característica individualista de los derechos anteriores, generando el reconocimiento de los derechos colectivos de distintos pueblos y culturas (Colpari, 2012; Ramírez, 2013). Además el proceso de globalización está produciendo la aparición de realidades que transforman profundamente la práctica de la ciudadanía; el fenómeno de la migración junto con las nuevas tecnologías son responsables de realidades de "ciudadanía transnacional", ciudadanos de una nación en la que no residen o de la que se encuentran temporalmente desplazados (Ramírez, 2013).

En todos estos cambios la participación ciudadana se está convirtiendo en una importante clave al ser considerada uno de los requisitos fundamentales y constitutivos de la gobernabilidad (Puelles y Urzúa, 1996). Podremos distinguir, no obstante, dos tipos básicos de participación si atendemos al problema que trata de resolver (Ibarra, 2008). Si persigue eliminar la falta de soberanía operativa individual o grupal hablaremos de una participación por "irrupción"; en cambio, si persigue la legitimación de determinadas instituciones, hablaremos de una participación por "invitación".

Mientras que esta última, acompañada de reformas "desde arriba", acaba generando una participación acotada y encauzada (Sebastiani, 2014), en los procesos de "irrupción" los movimientos sociales están realizando una función de resistencia a la pérdida de derechos así como de reivindicación de derechos colectivos. La ciudadanía activa pasaría de esta forma por la recuperación del espacio político por parte de los ciudadanos y ciudadanas (Mouffe, 1999).

Por ello, la necesidad de investigar la práctica ciudadana no solo se fundamenta en el cambio constante y cada vez más acelerado de la misma, sino también en que la participación activa se está convirtiendo en una herramienta política fundamental en el momento actual para la búsqueda de la emancipación social. Además, desde una perspectiva educativa, resulta imprescindible descubrir cómo se aprende y enseña a ejercer la ciudadanía, cómo se transforma y se transmite por los grupos que la ejercen, para poder desarrollarla en distintos contextos educativos.

La pregunta que se nos presenta llegado este punto es ¿desde qué posición epistemológica afrontamos la investigación de la práctica ciudadana? ¿Qué papel juegan la diversidad y la desigualdad, los procesos de inclusión y exclusión, en el ejercicio de la ciudadanía? En las siguientes líneas esbozamos una respuesta a esta pregunta, siempre limitada y en grado de tentativa, que parte de dos aproximaciones distintas, pero que a nuestro entender pueden resultar complementarias: la interseccionalidad y la interculturalidad. Antes presentamos brevemente una aproximación a la categorización, como contexto previo.

\section{El peligro categorizador. Diversidad, diferencia y desigualdad}

En nuestra sociedad, la exclusión sistemática de algunas personas y grupos (inmigrantes "ilegales", gitanos, personas sin hogar, etc.) se ha convertido en algo "normal" a lo que nos hemos acostumbrado, por lo que resulta en cierto modo invisible. Por lo general no se trata, como en otras épocas de la historia de la humanidad, de exclusiones radicales (expulsión, reclusión, ..., aunque es cierto que esto también sucede, como es el caso de algunos inmigrantes que son "devueltos" a su país), sino de considerar que estos grupos sociales tienen un estatus diferente que los convierte en subciudadanos.

Habitualmente se confunde diversidad cultural con categorización social (Aguado et al., 2010:37). Así, tradicionalmente se habla de las diferencias culturales en función de la etnia, lengua, nacionalidad, medio rural o urbano, o religión, aunque también se dan diferencias culturales entre los grupos de distintas edades, los distintos géneros, las diferentes clases sociales o económicas, etc. Las diferencias se enfatizan y se clasifican en grupos culturales o sociales definidos a priori, a los que se adscribe a las personas en función de su diferencia. Se homogeneiza, por un lado, a las personas que integran un grupo, y se jerarquiza, por otro lado, a los grupos y a las personas adscritas a ellos, de acuerdo con un patrón de valoración que sitúa en diferentes posiciones sociales a unos grupos y otros. 
El establecimiento de categorías sociales degenera en desigualdad por la valoración diferente de los grupos (jerarquización) y sitúa a unos y otros en posiciones privilegiadas o desfavorecidas respecto de los recursos, el poder o la consideración social. Los derechos de unos se enfrentan a los de los otros, y se naturaliza la desigualdad basándose en la diferencia (Grupo Inter, 2007:54).

La propia categorización es un acto de poder.

En el ámbito de la diversidad cultural y educación, buena parte de investigaciones responden a planteamientos iniciales que vinculan diversidad cultural con categorías previas ligadas al origen geográfico, en respuesta a los cambios demográficos de los últimos años.

\section{El análisis interseccional}

En una primera aproximación sería fácil observar el hecho de que la práctica de la ciudadanía se ve influida en muchos casos por el diferente acceso a oportunidades económicas y sociales; por ello parece que habría que tener en cuenta para su estudio las categorías sociales y culturales de las que dependen estas oportunidades, como pueden ser la "raza", la clase, el género, etc. No parece por tanto posible "pensar la ciudadanía sin tener en cuenta tales categorías" (Amparo Alves et al., 2014:6).

Para el estudio de diversas categorías sociales de privilegio y opresión y su interrelación, el análisis interseccional emerge como una de las mejores opciones. Fue Kimberlé Crenshaw quien acuñó el término Interseccional para poner de manifiesto cómo las luchas feministas o antirracistas podían producir el efecto perverso de aumentar la opresión en los casos en que distintos ejes de diferenciación social operaban conjuntamente, como en el caso de las mujeres negras (Crenshaw, 1991). Planteando además que la falta de visión interseccional en las políticas y reivindicaciones antirracistas y feministas proporcionan espacios no impugnados que pueden convertirse en debilidad de ambos movimientos (Crenshaw, 2012). Pero, a pesar de que la interseccionalidad se ha popularizado incorporándose incluso a foros internacionales, el término sigue siendo polisémico y controvertido.

En los análisis interseccionales se han distinguido diversos acercamientos (McCall, 2005; Yuval-Davis, 2013):

- los acercamientos Intercategoriales se concentrarían en la forma en que las distintas categorías sociales se relacionan entre sí. Estas categorías pueden ser construidas a priori aunque de manera provisional, y permiten el análisis multigrupal y comparativo así como acercamientos metodológicos estadísticos. Centran su interés en la relación entre las diversas categorías y los distintos grupos sociales que estas categorías definen, lo que hace que sean criticados porque pueden asumir un enfoque aditivo de las categorías, y porque la propia categorización es vista como sospechosa al simplificar la complejidad de la realidad social y producir exclusiones que pueden conducir a la desigualdad.

- En el otro extremo se situaría el acercamiento Anticategorial que, en línea con las críticas anteriores y con posiciones posmodernas, trata de deconstruir las categorías como forma de deconstruir las desigualdades, y propone por tanto eliminar las categorías sociales como único camino posible para suprimir la opresión en la sociedad (McCall, 2005). Desde este planteamiento la realidad social no encaja perfectamente en ninguna construcción categórica si no es por la imposición de un orden previo homogeneizante. Teniendo en cuenta además que las categorías sociales han sido utilizadas tradicionalmente para naturalizar y esencializar las diferencias legitimando la desigualdad (Olmos y Rubio, 2014).

- Entre uno y otro se encontraría el acercamiento Intracategorial que problematiza el significado y los límites de las mismas categorías, a la vez que trata de que las categorizaciones no sean producidas apriorísticamente, sino desde las realidades estudiadas. Tanto este acercamiento como el anterior suelen utilizar aproximaciones etnográficas y estudios de caso para comprender de manera compleja y densa la realidad estudiada, centrándose más en las realidades de un solo grupo o caso de estudio.

Como toda clasificación, esta no agota la realidad de las distintas investigaciones sobre la interseccionalidad, pues no todas podrían clasificarse en estos acercamientos y algunos casos cruzarían los límites de los mismos (McCall, 2005). A pesar de ello, y siguiendo a Antonia Olmos y María Rubio (2014), pueden asumirse unos mínimos comunes: - Hay múltiples formas de opresión y privilegio debido a la diversidad de categorizaciones socioculturales que las originan, las conexiones 
entre ellas y la dependencia contextual con la que funcionan.

- Existe diversidad de formas de experimentar esta diversidad de formas de privilegio y opresión.

- Esta diversidad de formas de opresión y privilegio son sustentadas por ideologías que esencializan las diferencias como forma de legitimar dicha desigualdad.

- Estas diversas categorías de diferenciación no funcionan de forma aditiva o multiplicativa, sino mutuamente constitutivas.

Por todo ello podemos entender la interseccionalidad como "los complejos, irreductibles, variados $\mathrm{y}$ variables efectos que resultan cuando múltiples ejes de diferencia -económica, política, cultural, psíquica, subjetiva y experiencial-se intersectan en contextos históricos específicos" (Brah y Phoenix ${ }^{4}$, citadas en Brah, 2013:14).

Desde esta definición podemos enfrentarnos al proceso de categorización entendiendo que las categorías no son intrínsecamente válidas, sino que parten de un conocimiento situado (Haraway, 1995). Es decir, en situaciones específicas en relación con personas específicas unas categorías serán más importantes que otras, aunque al mismo tiempo podamos encontrar algunos ejes de diferenciación o divisiones sociales que tienden a estar presentes de manera más habitual. Sin olvidar a su vez que en ningún caso son estáticas, estando siempre bajo continuos procesos de impugnación y cambio (Yuval-Davis, 2006).

\section{El enfoque intercultural}

Catherine Walsh (2010) propone, a modo sintético, distinguir entre tres perspectivas de la interculturalidad. Una relacional en referencia al contacto e intercambio entre culturas, cuyo problema puede residir en que puede minimizar y ocultar la dominación en la que se lleva a cabo la relación. Otra denominada funcional enraizada en el reconocimiento de la diversidad y la diferencia cultural con metas de inclusión de la misma en la estructura social establecida, y por tanto perfectamente compatible con la globalización neoliberal. Y la última, la interculturalidad crítica que parte del "reconocimiento de que la diferencia se construye dentro de una estructura y matriz colonial de poder racializado y jerarquizado $[\ldots \mathrm{y} \ldots]$ se entiende como una estratégica, acción y proceso permanentes de relación y negociación entre, en condiciones de respeto, legitimidad, simetría, equidad e igualdad" (Walsh, 2010:7879) constituyéndose como un proyecto político, social, ético y epistémico.

Esta propuesta se alinea con la pedagogía crítica, que se centra tanto en el desarrollo de la conciencia crítica individual con relación a las condiciones sociales injustas, como en el desarrollo de un componente colectivo de lucha y compromiso político para transformar dichas condiciones sociales injustas y crear una sociedad más igualitaria. Se trata de desarrollar en el alumnado la libertad (componente individual) solidaria (componente colectivo), y de hacerlo en el contexto educativo y en el contexto social más amplio. El desarrollo de la conciencia crítica individual se considera paso previo para la acción colectiva y la transformación social. Desde el enfoque intercultural resulta imprescindible superar la visión de la diferencia y la desigualdad como asunto privado, individual y que culpabiliza a la víctima de las injusticias sociales que padece. (Gil-Jaurena, 2008).

La ciudadanía participativa, crítica y transformadora (Mata-Benito et al., 2012 y 2013) que nos proponemos investigar entronca con la propuesta crítica de Walsh, teniendo en cuenta además que el enfoque intercultural acentúa la interacción, comunicación, negociación y enriquecimiento entre diversas prácticas culturales (Aguado, 2003), poniendo el foco en el contexto intersubjetivo donde se define la identidad y la cultura, producto de relaciones y razonamientos (Abdallah-Pretceille, 2006) y donde se da sentido y se construye la ciudadanía.

El acercamiento culturalista o multiculturalista y su riesgo de reificación de las diferencias y los grupos desde su énfasis categorizador ha sido contestado desde el enfoque intercultural. Así, frente a una idea homogénea y estática de la diversidad y la diferencia, la perspectiva intercultural presenta diversidad e igualdad como conceptos relacionales, y por ello, de ser vistas como un problema se convierten en oportunidades educativas, ya que "las diferencias culturales, étnicas, raciales e ideológicas incrementan la posibilidad de diálogo, confianza y solidaridad" (Giroux, 1992).

Podemos entender que, frente al culturalismo y la lógica de la diferencia propios del enfoque multicultural (Abdallah-Pretceille, 2001), el paradigma intercultural establece una lógica de la diversidad, capaz de proyectarse propositivamente hacia utopías de convivencia en o entre la diversidad y la igualdad (Gil-Jaurena, 2008). 
La lógica de la diferencia se inscribe en el marco de una lógica monódica que aísla las entidades desde el punto de vista de una relación no igualitaria. La diferencia no se manifiesta en el plano de las realidades, sino en el de los símbolos. La diferencia legitima la distancia, incluso el rechazo (Abdallah-Pretceille, 2001:20).

Así la apuesta del enfoque intercultural es:

- Volver a la idea original de "diversidad" (la diversidad como norma), abstrayéndonos de las categorías de diferencia preestablecidas que nos posicionan ante la realidad de manera prejuiciosa.

- Reconocer al mismo tiempo el valor de la diferenciación, que nos ayuda en nuestro primer acercamiento a la realidad y de hecho es la manera en que nos situamos frente al mundo, pero estar prevenidos sobre la simplificación que acarrea dicha categorización previa.

- Ser conscientes de los procesos de jerarquización de las diferencias, por los que "valoramos las cosas como somos, no como son".

- Anticipar y prevenir los efectos perversos de la categorización y valoración de la diversidad, que pueden llegar a esencializar y desvirtuar rasgos que en muchos casos no son tan relevantes, y a ocultar otros que sí lo son (Gil-Jaurena, 2008).

\section{Conclusión: Complementariedad de perspectivas para el análisis de la participación ciudadana}

Leyendo los epígrafes anteriores se puede interpretar que la única forma de que la interculturalidad y la interseccionalidad fueran complementarias sería desde la aproximación anticategorial, debido a que para ambas posturas la categorización es problemática por su tendencia reificadora y esencializadora de las diferencias. Para la perspectiva intercultural "reconocer la diversidad cultural implica reconocer al otro, huyendo de adscripciones previas, fijas y categorizadoras" (Aguado, 2009:17).

Desde este punto de vista, los peligros de la interseccionalidad -en su acercamiento intercategorial principalmente, pero también en el intracategorialpueden ser:

- Que la categorización impida ver la complejidad social real. Una complejidad en la que los ejes de diferenciación no funcionan individualmente, sino de forma conjunta y no todos tienen la misma importancia en cada contexto.
- Que las propias categorías de análisis establecidas acaben construyendo la diferencia, sirviendo de legitimación a ideologías que tratan de naturalizar o esencializar la desigualdad

- Que las categorías sean construidas apriorísticamente lo que no tiene por qué coincidir con las divisiones sociales de cada contexto sociohistórico, lo que, de nuevo impida ver la realidad social.

- Que se entiendan las relaciones categoriales de manera sumativa en vez de mutuamente constitutivas de situaciones complejas y únicas de privilegio y opresión, situaciones además que no son solo individuales y grupales, sino que se relacionan y comunican con otras generando cambio y transformaciones en dichas situaciones.

Por su parte la perspectiva intercultural puede tener sus puntos débiles principalmente en:

- El "olvido" consciente de las categorías establecidas a priori puede conllevar el ocultamiento precisamente de aquello que se quiere confrontar: la desigualdad de raíz y los procesos consecuentes de inclusión o exclusión social. Por tanto el anclaje anticategorial que se asume para evitar el acto de poder de la imposición de categorías como forma de eliminación de las desigualdades puede paradójicamente perpetuarlas al invisibilizarlas.

- La eliminación de las categorías puede hacer que las propuestas que se realicen no ayuden a alcanzar ese ideal perfectivo al no partir de una realidad en donde esas categorías no se han eliminado.

- Que aun en los casos en que se parta de planteamientos críticos que tratan de evidenciar las situaciones de opresión y privilegio -como en la propuesta de Walsh- el foco se restrinja a aspectos étnicos, olvidando otros ejes de diferenciación que pueden ser igual o más importantes en contextos concretos.

Ahora bien, una propuesta complementaria permitiría superar estas amenazas; si bien la interseccionalidad evitaría el "olvido" categorial de la interculturalidad, esta aportaría un objetivo emancipador que evitaría el peligro reificador de las categorías. De igual manera, la interculturalidad -al poner el foco en la comunicación e interrelación- evitaría el anclaje individual y grupal de la interseccionalidad y obligaría a situar las categorías y entenderlas de forma dinámica. La interseccionalidad, por su 
parte, aporta un punto concreto de partida para el objetivo transformador de la interculturalidad; y ambas ampliarían el foco de análisis de la otra aumentando la complejidad del mismo.

Por ello, para estudiar la práctica ciudadana, parece interesante utilizar las categorías sociales que la influyen, pero siempre desde la construcción y categorización que hacen las propias personas inmersas en el ejercicio ciudadano. Pues aunque el proceso de categorización es en sí un ejercicio de poder, no es un proceso unilateral, todos los actores implicados participan del proceso de categorización e identificación (Crenshaw, 1995). Y este proceso existe, por lo que no prestarle atención solo favorecería su invisibilización en vez de su aprensión crítica.

En trabajos previos del grupo de investigación en el que participamos ${ }^{5}$ se aprecia la evolución desde posiciones epistemológicas enmarcadas en un acercamiento que podemos denominar intercategorial o culturalista (como Aguado et al., 1999) a un progresivo movimiento anticategorial, agudizado por la necesidad de deconstruir las categorías ligadas al origen nacional (y asociadas a déficits o problemas) que hemos ido encontrando en diferentes investigaciones en torno a la diversidad cultural y la educación (Aguado et al., 2010; Gil-Jaurena, 2008). Sin embargo, la omnipresencia de las categorías nos conduce a plantear la conveniencia de complementar un enfoque intercultural crítico y desencializador con un análisis interseccional que, de la misma manera, entienda los mecanismos de diferencia y desigualdad con la complejidad necesaria.

De este modo, lejos de considerar ambos planteamientos como irreconciliables (por su posición ante las categorías), planteamos que una complementariedad de los mismos permitiría un mejor y más complejo análisis y una comprensión más profunda del ejercicio ciudadano contemporáneo.

\section{Referencias Citadas}

Abdallah-Pretceille, M.

2001 La educación intercultural. Barcelona: Idea Books. 2006 "Lo intercultural como paradigma para pensar la diversidad". Conferencia en el Congreso Internacional de Educación Intercultural. Formación del profesorado y práctica escolar. del 15 al 17 de Marzo. Madrid: UNED.

Aguado, T.

2003 Pedagogía Intercultural. Madrid: McGraw-Hill.

2009 "El enfoque intercultural como metáfora de la diversidad en educación". En Aguado, T. y Del Olmo, M. (Ed.). Educación Intercultural perspectivas y propuestas (pp. 13-27).

Aguado, T. (dir.); Gil, J. A.; Jiménez, R. A.; Sacristán, A.; Ballesteros, B.; Malik, B.; Sánchez, Mª F.

1999 Diversidad cultural e igualdad escolar. Un modelo para el diagnóstico y desarrollo de actuaciones educativas en contextos escolares multiculturales. Madrid: CIDE-Ministerio de Educación y Ciencia.

Aguado, T. (coord.).

2010 Diversidad cultural y logros de los estudiantes en educación obligatoria. Lo que sucede en las escuelas. Madrid: MEC-UNED. Estudios del CREADE N ${ }^{\circ} 4$.

Amorós, C.

2008 "El legado de la Ilustración: de las iguales a las idénticas". En A. H. Puleo (Ed.) El reto de la igualdad de género. Nuevas perspectivas en Ética y Filosofía Política (pp. 45-61). Madrid: Biblioteca Nueva.

Amparo Alves, J.; Moreno, V.; Ramos, B.

2014 Notas preliminares para un análisis interseccional de la violencia en el distrito de Aguablanca (Cali-Colombia). Colombia. Universidad Icesi, serie documentos de trabajo de CIES, $N^{\circ} 5$.

Benhabib, S.

2006 Las reivindicaciones de la cultura. Igualdad y diversidad en la era global. Buenos Aires: Katz.
Brah, A.

2013 "Pensando en y a través de la Interseccionalidad". En Zapata, M.; García, S. y Chan, J (eds.) La interseccionalidad a debate. Actas del congreso internacional "indicadores intersccionales y medidas de Inclusión social en instituciones de Educación Superiror. Instituto de estudios Latinoamericanos, Berlín, pp. 14-20.

Cabrera, F.

2002 Hacia una nueva concepción de la ciudadanía en una sociedad multicultural. En M. Bartolomé. (coord.) Identidad y Ciudadanía. Un reto a la Educación Intercultural. Madrid. Narcea ediciones. Pp. 79-104.

Colpari, O.

2011 "La nueva participación ciudadana en Ecuador y Bolivia ¿Resultados de la lucha del movimiento indígena-campesino?" Nómadas. Revista Crítica de Ciencias Sociales y Jurídicas. Pp. 141-153.

Crenshaw, K.

1991 "Mapping the Margins: Intersectionality, Identity Politics, and Violence against Women of Color". Stanford Law Review , 43(6), 1241-1299.

1995 "The identity factor in Multiculturalism". Liberal Education, 81(4), 6-11.

2012 "From private violence to mass incarceration: Thinking intersectional about women, race and social control". Ucla Law Review, 59, 1418-1472.

Gil-Jaurena, I.

2008 El enfoque intercultural en educación primaria: una mirada a la práctica escolar. Tesis doctoral. http://e-spacio. uned.es/fez/view.php?pid=tesisuned:Educacion-Igil

Giroux, H. A.

1992 "La enseñanza, la alfebetización y la política de la diferencia". En H.A. Giroux y R. Flecha. Igualdad educativa y diferencia cultural. Esplugues de Llobregat, El Roure. Pp. 11-26. 
Grupo Inter.

2007 Racismo: qué es y cómo se afronta. Una Guía para hablar de racismo. Madrid: Pearson.

Haraway, D. J.

1995 "Conocimientos situados: la cuestión científica en el feminismo y el privilegio de la perspectiva parcial", en Haraway, D. J. Ciencia, cyborg y mujeres. La reinvención de la naturaleza. Madrid: Cátedra, 313-346.

Ibarra, $\mathrm{P}$.

2008 "Participación y poder: de la legitimación al conflicto." En Ibarra, P y Ahedo, I. Democracia Participativa y desarrollo humano. Madrid. Dykinson. Pp. 37-56.

Irurozqui, $\mathrm{M}$.

2008 "El mundo no es ancho ni ajeno. Nociones básicas sobre la ciudadanía y una propuesta conceptual sobre el valor público de la infracción electoral". En J.A. Téllez (coord.). Educacion Intecultural. Miradas multidisciplinares. Madrid. Los libros de la Catarata. Pp. 151-187.

Kymlicka, W.

1999 "Nacionalismos minoritarios dentro de las democracias liberales". En S. García y S. Lukes (comps.), Ciudadanía: justicia social, identidad y participación (pp. 127-157). Madrid: Siglo XXI.

Marshall, T. H.

[1949]1997 Ciudadanía y Clase Social. REIS. Revista española de investigaciones sociológicas (79), 297-344.

Mata-Benito, P.; Ballesteros-Velázquez, B.; Padilla-Carmona, M.

2012 "Ciudadanía crítica, participativa y transformadora: experiencias de aprendizaje" en de Alba Fernández, N.; García Pérez, F. y Santiesteban Fernández, A. (eds.) Educar para la participación en la enseñanza de las Ciencias Sociales, Vol. 2. Sevilla: Asociación Universitaria de Profesores de Didáctica de las Ciencias Sociales y Diada Editora. Pp. 131-138.

2013 "Ciudadanía Participativa y Transformadora: Análisis de Discursos y Propuestas de Aprendizaje". Revista Teoría de la Educación, 25(2), Pp. 49-68.

McCall, L.

2005 "The Complexity of Interseccionality". Signs, 30(3), 1771-1800. http://dx.doi.org/10.1086/426800

Mouffé, C.

1999 El retorno de lo político. Barcelona: Paidós.

Olmos, A.; Rubio, M.

2014 "Imaginarios sociales sobre 'la/el buen y la/el mal estudiante': Sobre la necesidad de un análisis interseccional para entender las lógicas de construcción de la diferencia hacia el alumnado 'inmigrante"”. en Cucalón, P. (Ed.). Etnografía de la escuela y la interseccionalidad. Madrid: Traficantes de sueños.
Pateman, C.

1996 "Críticas feministas a la dicotomía público/privado". En

C. Castells (ed.), Perspectivas feministas en teoría política (pp. 31-52). Barcelona: Paidós.

Procacci, G.

1999 Ciudadanos pobres, la ciudadanía social y la crisis de los estados del bienestar. En Soledad García y Steven Lukes (comps.), Ciudadanía: justicia social, identidad y participación (pp. 15-44). Madrid: Siglo XXI.

Puelles, M.; Urzúa, R.

1996 "Educación, gobernabilidad democrática y gobernabilidad de los sistemas educativos" en Revista Iberoamericana de Educación, № 12. Pp. 107-136.

Ramírez, A.

2013 “Ciudadanías étnicas y/o plurales en el contexto actual boliviano?" Arqueoantropológicas. Año 3, Nº 3. Pp. 153-183.

Sassen, S.

2001 ¿Perdiendo el control? La soberanía en la era de la globalización. Barcelona: Bellaterra.

Somers, M. R.

1999 La ciudadanía y el lugar de la esfera pública: un enfoque histórico. En Soledad Garcia y Steven Lukes (comps.), Ciudadanía: justicia social, identidad y participación (pp. 217-234). Madrid: Siglo XXI.

Suárez Navaz, L.

1998 "Fronteras y ciudadanía: nuevos desafíos de un viejo modelo desde una perspectiva antropológica”. En E. Martín Díaz y S. de la Obra Sierra, Repensando la Ciudadanía (pp. 167-200). Sevilla: Fundación El Monte.

Walsh, C.

2010 "Interculturalidad crítica y educación intercultural", en Viaña, Jorge; Tapia, Luis y Walsh, Catherine, Construyendo interculturalidad crítica, La Paz, Instituto Internacional de Integración, Convenio Andrés Bello, pp. 75-96.

Young, I.M.

1996 "Vida política y diferencia de grupo: una crítica del ideal de ciudadanía universal”. En C. Castells (Ed.) Perspectivas feministas en teoría política (pp. 99-126). Barcelona: Paidós. Yuval-Davis, $\mathrm{N}$.

2006 "Intersectionality and Feminist Politics". European Journal of Women's Studies, 13. Pp. 193-209.

2013 "Más allá de la dicotomía del reconocimiento y la redistribución. Interseccionalidad y estratificación" En Zapata, M., García, S. y Chan, J (eds.) La interseccionalidad a debate. Actas del congreso internacional "indicadores interseccionales y medidas de Inclusión social en instituciones de Educación Superior. Instituto de estudios Latinoamericanos, Berlín. Pp. 21-34.

\section{Notas}

1 La aproximación evolucionista de Marshall ha sufrido infinidad de críticas, Procacci (1999) critica su anglocentrismo y que no tenga en cuenta la realidad francesa, Somers (1999) critica su anclaje a la existencia de determinadas clases sociales, etc. Irurozqui (2008) hace un breve repaso al desarrollo histórico de los estudios sobre la ciudadanía muy interesante.

2 La cita de Cabrera se refiere a Babiano, J. (2000). Ciudadanía y exclusión. En Pérez, M. (comp.) Ciudadanía y Democracia (Pp. 237-295). Madrid: Largo Caballero.
3 Como puede desprenderse de noticias e informes sobre tortura y represión, valga como muestra el último informe de Amnistía Internacional "La tortura en 2014. 30 años de promesas incumplidas" http://www.amnesty.org/en/ library/asset/ACT40/004/2014/ar/43dfeb4e-2e43-47e6abef-900b54756684/act400042014es.pdf

4 La cita de Avtar Brah se refiere a Brah, A. and Phoenix, A. (2004) "Ain't I a woman? Revisiting Intersectionality". Journal of International Women's Studies, 5(3). Pp. 75-86.

5 Grupo INTER de investigación en educación intercultural. www.uned.es/grupointer 
